\title{
Vibration control of linear split Stirling cryogenic cooler for airborne infrared application
}

\author{
A.M. Veprik ${ }^{\mathrm{a}, *}$, V.I. Babitsky ${ }^{\mathrm{a}}$, N. Pundak ${ }^{\mathrm{b}}$ and \\ S.V. Riabzev ${ }^{\mathrm{b}}$ \\ ${ }^{a}$ Department of Mechanical Engineering, \\ Loughborough University, Loughborough, \\ Leicestershire, LE11 3TU, UK \\ Tel.: +44 1509 223177; Fax: +44 1509223934 \\ ${ }^{\mathrm{b}} R \& D$ Department, RICOR, Cryogenic and Vacuum \\ Systems, Kibbutz En Harod Ihud, 18986, Israel \\ Tel.: +9726 6530770; Fax: +9726 6532424; E-mail: \\ S.Riabzev@ricor.com
}

Received 18 February 2000

Revised 24 July 2000

Modern infrared imagers often rely on the split Stirling cryogenic coolers the linear compressors of which are the wellknown sources of harmonic disturbance. The traditional method of their passive isolation fails to meet the restraints on the static and dynamic deflections which are originated by the combined action of the airborne g-loading and harsh random vibration.

The vibration protection system, which combines a stiff and heavily damped vibration isolator with tuned dynamic absorber, is studied and optimised for use in the design of an airborne infrared device.

Such a design is aimed, primarily, at essential dynamic suppression of the harmonic force which is produced by the linear compressor and, secondarily, at minimisation of environmental vibration loads transmitted through the infrared device to the linear compressor. Experimental testing backed up the theoretical results.

\section{Introduction}

Infrared (IR) imagers enhance tremendously the ability to detect and track ground, sea and air targets and also to navigate at nighttime $[3,5]$. Their operating

\footnotetext{
*Corresponding author; E-mail: A.Veprik@lboro.ac.uk.
}

principle is based on that simple fact that warmer objects radiate more and cooler objects radiate less. Since the noise figure of an IR sensor strongly depends on its operating temperature, the high-resolution imager requires cryogenic cooling down to $80 \mathrm{~K}$ and a high level of optical stabilisation.

Modern sophisticated thermal imagers often rely on the closed cycle split Stirling cryogenic cooler. Such a cooler comprises two separate components: linear compressor and expander. These are interconnected by a flexible gas transfer line (thin-walled stainless steel tube of small diameter) to provide maximum flexibility in system design and to isolate the IR detector from vibration interference. The compressor unit is located in a sealed casing and typically contains a reciprocating piston compressor which is driven harmonically by a linear motor. This provides the required pressure pulses and the volumetric reciprocal change of the working agent (helium) in the expansion space of the expander unit. The expander unit is also located in the sealed casing and typically contains a pneumatically driven displacer-regenerator. The displacer shuttles the working agent back and forth from the cold side to the warm side of the cooler. During the expansion stage of the thermodynamic cycle, heat is absorbed from the IR sensor which is thermally connected to the cold side, and during the compression stage heat is rejected at the warm side [5,12]. Figure 1 shows the RICOR model K529 split Stirling cryogenic cooler which is optimised to produce $1 \mathrm{~W}$ heat lift at $80 \mathrm{~K}$. Typical images of Integrated Detector Dewar and Cooler Assembly for military scanning applications which rely on such a cooler may be found, for example, in Sofradir Website. ${ }^{1}$

Since such a compressor is of a single-piston design, the reciprocating motion of its unbalanced parts produces significant level of vibration interference, or

\footnotetext{
${ }^{1}$ See http://infrared.sofradir.com/pages/products/pg standard mot.
} htm. 


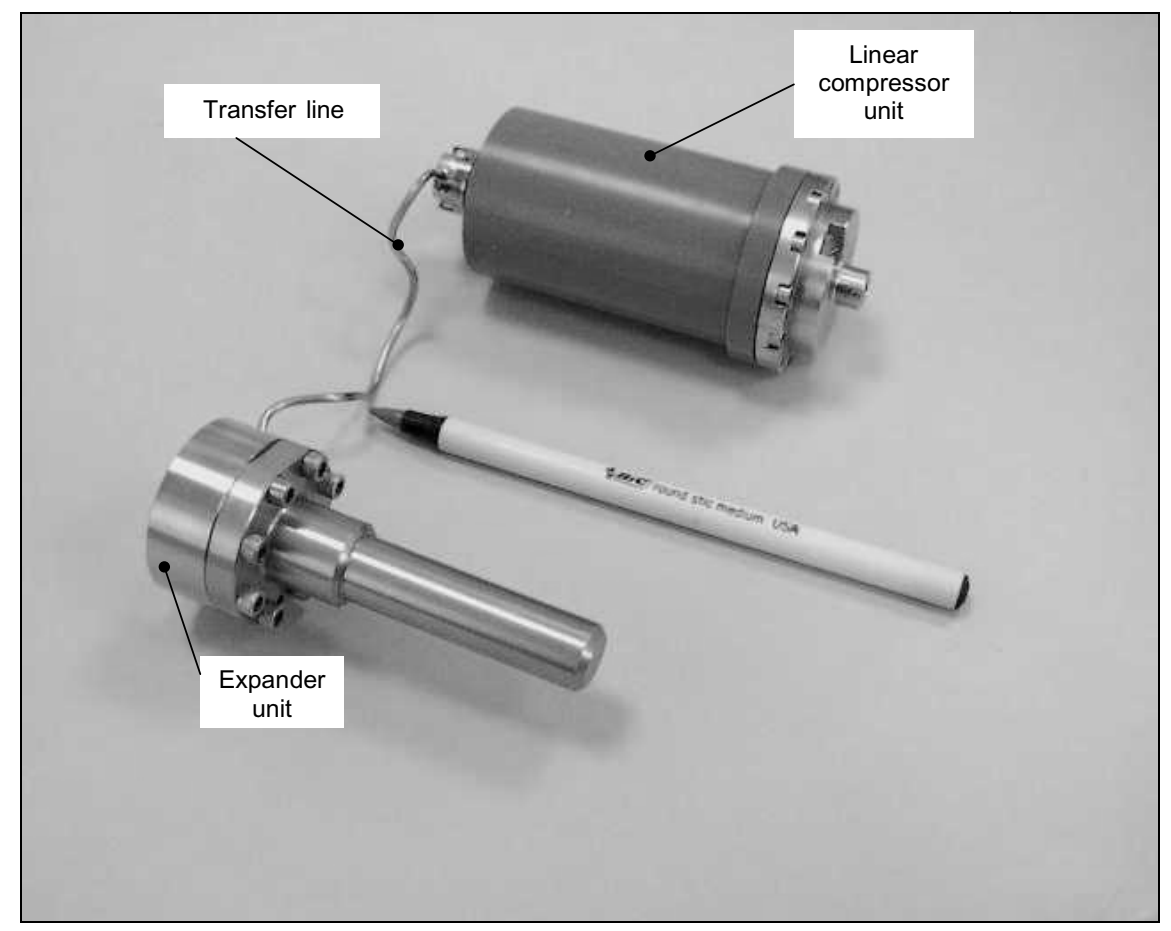

Fig. 1. Ricor model K529 linear split Stirling cryogenic cooler.

self-induced force as it is described in the practice of airborne design. Such forces may cause harmful relative deflection of the IR sensor to the rest of the optical system. This in particular holds true for such optical or electro-optical systems where all elements can not be mounted on a single rigid integrated structure (optical bench). The excessive level of the line-of-sight jitter (as compared with spatial resolution of the particular IR sensor) may cause drastic degradation in quality of the IR image. The suppression of these forces, therefore, becomes imperative.

At the same time, the airborne IR instrumentation is typically subjected to the combined action of harsh environmental vibration and shocks. Since the endurance of the cooler is also a concern, the substantive attenuation of the severity of random vibration transmitted to the linear compressor through the vibration of the IR device is desirable.

Vibration isolation is the simplest, most widespread and well-studied method of vibration protection [9-11]. It involves application of the resilient mounting which provide for the desired attenuation. As is known, the best isolation in the typical high frequency span may be achieved when the natural frequency and loss factor of the vibration isolator are low. Unfortunately, such an isolator is only feasible in such applications where the intensive shock, random excitation and constant accelerations (g-loads) are not typical.

As the equipment containing such a low frequency and lightly damped vibration isolator involves an exposure to the real world harsh environmental conditions, which are typical for airborne applications, the problem of excessive deflections becomes a serious concern. These conditions typically encounter wideband random excitation (e.g. flight through turbulent flow) and high g-loads which are experienced by the airborne vehicle at take-off, climb, high-speed turn, speedup, etc.

As a result, plenty of free "rattle space" must be allowed around the equipment and also the vibration isolators, thermal and electrical interfaces need to be of special design. Such an approach typically complicates the entire design and makes it both unreliable and costineffective.

The demand on compactness of the whole package, along with the smallness of deformations of the gas transfer line, thermal and electrical interfaces impose particular limitations on the allowed relative rattling of the components of the cryogenic cooler.

In $[1,2,8]$, these objectives are achieved by mounting the linear compressor upon the lightly damped and low frequency vibration isolators providing for desired 
attenuation of the self-induced force. The problem of excessive deflections under external severe wideband random vibration and shock was successively solved by means of the visco-elastic bumpers which were installed with minimal possible rattle-space and designed to perform as an optimal shock absorber. The above solution, however, appears to be suitable for $1 \mathrm{~g}$ environments only (e.g. tracked vehicles, marine and ground applications). In spite of the fact that high gloads (which are typical for airborne applications only) do not overload an application dynamically, the excessive quasi-static deflections may cause a lockup in the system of bumpered vibration protection resulting in the complete degradation of vibration isolation. This may become an important concern because the action of such high g-loads may be a long-term event and the system of vibration protection, evidently, must be free of malfunctioning during these events.

An increase in the natural frequency and loss factor of an isolator allows for the close control of the above excessive deflections but evidently negates the efficiency of the vibration isolation in the typical high-frequency span. On occasion it becomes impossible to trade off the limitations imposed on the allowable deflections of the linear compressor with the required vibration attenuation at the driving frequency. Thus, the principle of linear vibration isolation becomes inadequate.

Accounting for the combined action of high g-loads, harsh wideband random vibration and intensive harmonic self-induced force requires an entirely different approach to the design of vibration protection system for linear compressor.

Ideally, such a vibration protection system has to provide for:

- high static stiffness,

- high dynamic stiffness at all frequencies,

- low dynamic stiffness at the discrete disturbance (driving) frequency.

Traditionally, such a combination is achieved by the active adaptive design. For example, CSA Engineering ${ }^{2}$ proposes the "Adaptive Vibration Suppression Mount for Cryogenic Coolers" which is capable of embedding the controllable "notches" in the frequency response of vibration protection system by a proper combination of active piezoceramics and passive isolators.

In this article, the authors examine the idea of utilisation of the tuned dynamic absorber in combination with

\footnotetext{
${ }^{2}$ See http://sbir.gsfc.nasa.gov/SBIR/mall2.htm.
}

the stiff and heavily damped vibration isolator for the simultaneous dynamic suppression of the self-induced forces and the attenuation of the based-induced random vibration. It is shown that such an entirely passive system may be optimally designed to meet the above requirements.

The linear split Stirling cryogenic cooler is typically driven at constant frequency which may be easily and accurately tuned and maintained by means of the appropriate digital controller. Moreover, the dynamic force which is exported by such a compressor is, in general, axially directed. Therefore, the desired control of the self-induced harmonic force may be achieved by means of single tuned vibration absorber.

It is important to note that the use a resilient mounting is obligatory for the dynamic de-coupling from the base and for the normal performance of the tuned dynamic absorber. It is shown in the present article that the suppression index is reciprocal to the mass ratio [ absorber mass $]$. If the equipment which embodies the dynamic absorber is mounted rigidly to the base, the equipment mass will be apparently increased by the effective mass of the base. This would cause the drastic decrease in the mass ratio and, therefore, deterioration of the dynamic suppression index and also an enormous increase in the dynamic magnitude of the dynamic absorber.

It is also shown in the present article, that the suppression index is reciprocal to the loss factor in the dynamic absorber. Therefore, an application of the undamped tuned dynamic absorber is vital for efficient dynamic suppression at anti-resonant frequency. At the same time, this typically causes new problems due to the appearance of additional resonance [7,9], thus the dynamic response of the combined two degree of freedom (TDOF) system (primary system + dynamic absorber) might become excessive. This, in particular, holds true when the damping in the primary suspension is small. Snowdon [9] showed that the addition of the damping to the primary system might essentially control the above dynamic response at both resonances simultaneously. However, the influence of the above damping on the suppression at anti-resonant frequency has not yet been studied.

It is apparent from common sense that the damping in the primary suspension might be optimised. Really, if this damping tends to zero, the RMS response to random excitation tends to infinity [4]. Further, if the above damping is too high, the primary system appears to be virtually bonded to the moving base; hence, attenuation of wideband random vibration and also dy- 
namic suppression at anti-resonant frequency becomes impossible.

In the present article, the authors address the principles of optimal design of the system of vibration protection which combines stiff and heavily damped primary suspension with tuned vibration absorber for operation in the harsh airborne environments (wideband random vibration and high constant accelerations).

Based on the results of the solution obtained for the optimal problem, the system of vibration protection is designed for the particular IR application which relies on the RICOR split Stirling cryogenic cooler model K529 (as shown in Fig. 1).

The design objectives are as follows:

- suppression of the self-induced force at driving frequency by the factor of 20 ,

- maintenance of the peak deflection of the flexurally suspended compressor within allowable $\pm 1 \mathrm{~mm}$,

- maximal attenuation of the base-induced broadband vibration.

\section{Dynamic model of vibration protection system}

Figure 2 shows the generic dynamic model of the vibration protection system comprising a vibration isolator and dynamic absorber. The primary sub-system of mass $M_{1}$ which represents the linear compressor is suspended from the base by the vibration isolator which stiffness and damping are $K_{1}$ and $B_{1}$, respectively. The self-induced sinusoidal force, $F(t)=F_{0} \sin v t$, where $F_{0}$ and $v$ denote magnitude and frequency, is applied to the primary system. The motion of the support, $Y(t)$, comprises wideband random vibration and constant accelerations (g-load).

The dynamic absorber of mass $M_{2}$ is attached to the primary system through the flexural element the stiffness and damping of which are $K_{2}$ are $B_{2}$, respectively.

Since the linearity of the system is assumed, the principle of superposition becomes applicable. To define the dynamic response to different sources of excitation some basic relationships are detailed below.

\subsection{Dynamic response of TDOF system to random excitation}

We assume that the acceleration of the base, $\ddot{Y}(t)$, is a normally distributed wideband random process which is defined by the power spectral density (PSD) $S_{\ddot{Y}}(\omega)$, where $\omega \in[0, \infty[-$ angular frequency. We also assume now that in Fig. $2, F(t) \equiv 0$.

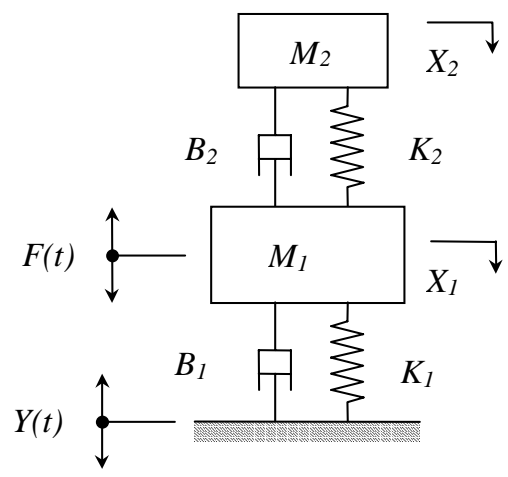

Fig. 2. Generic dynamic model of vibration protection system.

The following equations of motion are applicable for the model in Fig. 2:

$$
\begin{aligned}
& M_{1} \ddot{X}_{1}+K_{1} X_{1}+B_{1} \dot{X}_{1}+K_{2}\left(X_{1}-X_{2}\right) \\
& +B_{2}\left(\dot{X}_{1}-\dot{X}_{2}\right)=K_{1} Y_{1}+B_{1} \dot{Y}_{1} \\
& M_{2} \ddot{X}_{2}+K_{2}\left(X_{2}-X_{1}\right)+B_{2}\left(\dot{X}_{2}-\dot{X}_{1}\right)=0
\end{aligned}
$$

An application of the Laplace transform

$$
X_{1,2}(t) \Leftrightarrow x_{1,2}(s), Y(t) \Leftrightarrow y(s)
$$

yields the absolute transfer function of the primary system

$$
\begin{aligned}
T(s) & =\frac{x_{1}(s)}{y(s)} \\
& =\frac{\left(K_{1}+s B_{1}\right)\left(M_{2} s^{2}+K_{2}+s B_{2}\right)}{D(s)}
\end{aligned}
$$

where

$$
\begin{aligned}
D(s)= & {\left[M_{1} s^{2}+K_{1}+K_{2}+s\left(B_{1}+B_{2}\right)\right] } \\
& {\left[M_{2} s^{2}+K_{2}+s B_{2}\right]-\left(K_{2}+s B_{2}\right)^{2} }
\end{aligned}
$$

is the characteristic polynomial and $s$ is the complex (Laplace) variable.

The corresponding relative transfer function is:

$$
T_{\text {rel }}(s)=\frac{x_{1}(s)-y(s)}{y(s)}=T(s)-1,
$$

For the complex transmissibilities, after substitution $s=j \omega$, where $j=\sqrt{-1}$, we find:

$$
\begin{aligned}
& T(j \omega)= \\
& {\left[\left(K_{1}+j \omega B_{1}\right)\left(-M_{2} \omega^{2}+K_{2}+j \omega B_{2}\right)\right] /} \\
& \left\{\left[-M_{1} \omega^{2}+K_{1}+K_{2}+j \omega\left(B_{1}+B_{2}\right)\right]\right. \\
& \left.\left[-M_{2} \omega^{2}+K_{2}+j \omega B_{2}\right]-\left(K_{2}+j \omega B_{2}\right)^{2}\right\},
\end{aligned}
$$




$$
\begin{aligned}
& T_{r e l}(j \omega)=T(j \omega)-1=\omega^{2}\left[K_{2} M_{1}+K_{2} M_{2}\right. \\
& \left.-M_{1} M_{2} \omega^{2}+j \omega B_{2}\left(M_{1}+M_{2}\right)\right] / \\
& \left\{\left[-M_{1} \omega^{2}+K_{1}+K_{2}+j \omega\left(B_{1}+B_{2}\right)\right]\right. \\
& \left.\left[-M_{2} \omega^{2}+K_{2}+j \omega B_{2}\right]-\left(K_{2}+j \omega B_{2}\right)^{2}\right\} .
\end{aligned}
$$

The PSD of absolute accelerations and relative displacements are calculated in the well-known form [4, 11]:

$$
\begin{aligned}
& S_{\ddot{X}_{1}}(\omega)=|T(j \omega)|^{2} S_{\ddot{Y}}(\omega), \\
& S_{X_{1 r e l}}(\omega)=\frac{1}{\omega^{4}}\left|T_{r e l}(j \omega)\right|^{2} S_{\ddot{Y}}(\omega) .
\end{aligned}
$$

The variances of the resultant absolute acceleration and relative displacements of the primary system may be found by means of the formulae [4,11]:

$$
\begin{aligned}
& \sigma_{\ddot{X}_{1}}^{2}=\frac{1}{2 \pi} \int_{0}^{\infty}|T(j \omega)|^{2} S_{\ddot{Y}}(\omega) d \omega \\
& \sigma_{X_{1 r e l}}^{2}=\frac{1}{2 \pi} \int_{0}^{\infty} \frac{1}{\omega^{4}}\left|T_{r e l}(j \omega)\right|^{2} S_{\ddot{Y}}(\omega) d \omega .
\end{aligned}
$$

The wideband random test is specified in accordance with [6] as follows: flat PSD $0.7 \mathrm{~g}^{2} / \mathrm{Hz}$ in the frequency range 10-2000 Hz (14g RMS overall level). The "white noise" approximation, $S_{\ddot{Y}}(\omega)=S_{0}=$ const, is, therefore, applicable. The above integrals in this case take the form:

$$
\begin{aligned}
& \sigma_{\ddot{X}_{1}}^{2}=\frac{S_{0}}{2 \pi} \int_{0}^{\infty}|T(j \omega)|^{2} d \omega \\
& \sigma_{X_{1 \text { rel }}}^{2}=\frac{S_{0}}{2 \pi} \int_{0}^{\infty} \frac{1}{\omega^{4}}\left|T_{r e l}(j \omega)\right|^{2} d \omega
\end{aligned}
$$

and may be evaluated analytically with the use of tables [4] by assuming the practical case of small damping in the secondary subsystem: $B_{2}=0$. It is our intention to design the vibration absorber with the lowest possible loss factor in order to achieve better vibration suppression at anti-resonant frequency. From (1) and (2), the simplified expressions for appropriate transmissibilities take the form:

$$
\begin{aligned}
T(j \omega)= & {\left.\left[K_{1}^{2}+\omega^{2} B_{1}^{2}\right)\left(-M_{2} \omega^{2}+K_{2}\right)^{2}\right] / } \\
& \left\{\left[-M_{1} \omega^{2}+K_{1}+K_{2}+j \omega B_{1}\right]\right. \\
& {\left.\left[-M_{2} \omega^{2}+K_{2}\right]-K_{2}^{2}\right\} } \\
T_{r e l}(j \omega)= & \omega^{2}\left[K_{2} M_{1}+K_{2} M_{2}-M_{1} M_{2} \omega^{2}\right] / \\
& \left\{\left[-M_{1} \omega^{2}+K_{1}+K_{2}+j \omega B_{1}\right]\right. \\
& {\left.\left[-M_{2} \omega^{2}+K_{2}\right]-K_{2}^{2}\right\} . }
\end{aligned}
$$

With the use of tables [4] we find:

$$
\begin{aligned}
& \frac{1}{2 \pi} \int_{0}^{\infty}\left[K_{1}^{2}\left(-M_{2} \omega^{2}+K_{2}\right)^{2}\right] / \\
& \left\{\mid\left[-M_{1} \omega^{2}+K_{1}+K_{2}+j \omega B_{1}\right]\right. \\
& {\left.\left[-M_{2} \omega^{2}+K_{2}\right]-\left.K_{2}^{2}\right|^{2}\right\} d \omega } \\
= & \frac{K_{1}}{2 B_{1}}, \\
& \frac{1}{2 \pi} \int_{0}^{\infty}\left[\omega^{2} B_{1}^{2}\left(-M_{2} \omega^{2}+K_{2}\right)^{2}\right] / \\
& \left\{\mid\left[-M_{1} \omega^{2}+K_{1}+K_{2}+j \omega B_{1}\right]\right. \\
& {\left.\left[-M_{2} \omega^{2}+K_{2}\right]-\left.K_{2}^{2}\right|^{2}\right\} d \omega } \\
= & \frac{B_{1}}{2 M_{1}}, \\
& \frac{1}{2 \pi} \int_{0}^{\infty} \frac{1}{\omega^{4}}\left[\omega ^ { 4 } \left(K_{2} M_{1}+K_{2} M_{2}\right.\right. \\
& \left.\left.-M_{1} M_{2} \omega^{2}\right)^{2}\right] /\left\{\mid\left[-M_{1} \omega^{2}+K_{1}+K_{2}\right.\right. \\
& \left.\left.+j \omega B_{1}\right]\left[-M_{2} \omega^{2}+K_{2}\right]-\left.K_{2}^{2}\right|^{2}\right\} d \omega \\
& \frac{M_{1}^{2}}{2 B_{1} K_{1}}\left[1+2 \frac{M_{2}}{M_{1}}+\left(\frac{M_{2}}{M_{1}}\right)^{2} \frac{K_{1}}{K_{2}}\right. \\
& +. \\
& \\
&
\end{aligned}
$$

For the RMS of absolute acceleration and relative displacement of the primary system we obtain finally:

$$
\sigma_{\ddot{X}_{1}}=\sqrt{\frac{S_{0}}{2}\left(\frac{K_{1}}{B_{1}}+\frac{B_{1}}{M_{1}}\right)}
$$

and

$$
\begin{aligned}
& \sigma_{X_{1 \text { rel }}}= \\
& \sqrt{\frac{S_{0}}{2} \frac{M_{1}^{2}}{B_{1} K_{1}}\left[1+2 \frac{M_{2}}{M_{1}}+\left(\frac{M_{2}}{M_{1}}\right)^{2} \frac{K_{1}}{K_{2}}+\left(\frac{M_{2}}{M_{1}}\right)^{2}\right]}
\end{aligned}
$$

Further $\Omega_{i}=\sqrt{\frac{K_{i}}{M_{i}}}$ denotes partial natural frequencies, $\zeta_{i}=B_{i} / 2 M_{i} \Omega_{i}$ denotes the partial loss factors $(i=1,2)$ and $\mu=\frac{M_{2}}{M_{1}}$ denotes the mass ratio.

Substitution of these notations into (3) and (4) yields

$$
\sigma_{\ddot{X}_{1}}=\sqrt{\frac{S_{0} \Omega_{1}}{2}\left(\frac{1}{2 \zeta_{1}}+2 \zeta_{1}\right)},
$$




$$
\sigma_{X_{1 \text { rel }}}=\sqrt{\frac{S_{0}}{4 \Omega_{1}^{3} \zeta_{1}}\left[1+\mu\left(2+\frac{\Omega_{1}^{2}}{\Omega_{2}^{2}}\right)+\mu^{2}\right]}
$$

It is important to note that the variance of acceleration of the primary mass is independent of the parameters of the secondary system. It is also important to note that an increase in the mass ratio causes an increase in the variance of relative deflection of the primary system.

\subsection{Quasi-static response of TDOF system to g-load}

The quasi-static deflection due to the g-loading is:

$$
\lambda=\frac{G(1+\mu)}{\Omega_{1}^{2}} .
$$

where $G$ is the value of constant acceleration. Through the mass ratio this expression accounts for the presence of the secondary system.

\subsection{Vibration suppression at anti-resonance}

To calculate the vibration suppression index at antiresonance, we refer to Fig. 2 and consider that $\ddot{Y}(t) \equiv 0$ and $F(t) \neq 0$.

The following equations of motion are applicable:

$$
\begin{aligned}
& M_{1} \ddot{X}_{1}+K_{1} X_{1}+B_{1} \dot{X}_{1}+K_{2}\left(X_{1}-X_{2}\right) \\
& +B_{2}\left(\dot{X}_{1}-\dot{X}_{2}\right)=F(t), \\
& M_{2} \ddot{X}_{2}+K_{2}\left(X_{2}-X_{1}\right)+B_{2}\left(\dot{X}_{2}-\dot{X}_{1}\right)=0 .
\end{aligned}
$$

An application of the Laplace transform

$$
X_{1,2}(t) \Leftrightarrow x_{1,2}(s), F(t) \Leftrightarrow f(s)
$$

yields the absolute transmissibility which gives the relationship between the force applied to the primary system and the total force

$$
R(t)=K_{1} X_{1}(t)+B_{1} \dot{X}_{1}(t)
$$

which is transmitted to the base through the elastic and viscous components of the primary vibration isolator:

$$
\begin{aligned}
& T(s)=\frac{r(s)}{f(s)} \\
= & {\left[\left(K_{1}+s B_{1}\right)\left(M_{2} s^{2}+K_{2}+s B_{2}\right)\right] / } \\
& \left\{\left[M_{1} s^{2}+K_{1}+K_{2}+s\left(B_{1}+B_{2}\right)\right]\right. \\
& {\left.\left[M_{2} s^{2}+K_{2}+s B_{2}\right]-\left(K_{2}+s B_{2}\right)^{2}\right\}, }
\end{aligned}
$$

where $R(t) \Leftrightarrow r(s)$.

For the absolute complex transfer function we find by substituting $s=j v$ :

$$
\begin{aligned}
& T(j v) \\
= & {\left[\left(K_{1}+j v B_{1}\right)\left(-M_{2} v^{2}+K_{2}+j v B_{2}\right)\right] / } \\
& \left\{\left[-M_{1} v^{2}+K_{1}+K_{2}+j v\left(B_{1}+B_{2}\right)\right]\right. \\
& {\left.\left[-M_{2} v^{2}+K_{2}+j v B_{2}\right]-\left(K_{2}+j v B_{2}\right)^{2}\right\} . }
\end{aligned}
$$

By dividing both numerator and denominator by $M_{1} M_{2}$ we readily find:

$$
\begin{aligned}
& T(j v)= \\
& {\left[\left(\Omega_{1}^{2}+j 2 \zeta_{1} v \Omega_{1}\right)\left(-v^{2}+\Omega_{2}^{2}+j 2 \zeta_{2} v \Omega_{2}\right)\right] /} \\
& \left\{\left[-v^{2}+\Omega_{1}^{2}+\mu \Omega_{2}^{2}+j 2 v\left(\zeta_{1} \Omega_{1}+\mu \zeta_{2} \Omega_{2}\right)\right]\right. \\
& \left.\left[-v^{2}+\Omega_{2}^{2}+j 2 \zeta_{2} v \Omega_{2}\right]-\mu\left(\Omega_{2}^{2}+j 2 \zeta_{2} v \Omega_{2}\right)^{2}\right\},
\end{aligned}
$$

where the notations for mass ratio, partial natural frequencies and loss factors were defined previously.

For the small values of the loss factor, $\zeta_{2}$, the magnitude of the complex transmissibility, $T(j v)$, reaches the minimum at anti-resonance, i.e. when $v=\Omega_{2}$. By a designing such a dynamic absorber, we can closely control the force transmission to the base at this particular anti-resonant frequency. An analysis of the expression of transmissibility, $T(j v)$, indicates, as usual, the high sensitivity of its magnitude to small frequency variations. Hence, the accurate tuning and maintenance of the driving frequency becomes a concern.

The actual level of force transmission, $T_{0}$, must be assessed in terms of transmissibility at frequencies $v_{d} \pm$ $\Theta$, where $v_{d}$ is the nominal driving frequency, and $\Theta$ denotes maximal detuning given by the design of the digital controller, that is:

$$
T_{0}=\max \left\{\left|T\left[j\left(v_{d}-\Theta\right)\right]\right|,\left|T\left[j\left(v_{d}+\Theta\right)\right]\right|\right\} .
$$

In the close vicinity of the anti-resonant frequency $v \approx \Omega_{2}$ the expression for the complex absolute transmissibility (8) may be simplified, as follows:

$$
\begin{aligned}
& \left.T(j v)\right|_{v \approx \Omega_{2}} \\
= & -\frac{\left(\Omega_{1}^{2}+j 2 \zeta_{1} v \Omega_{1}\right)\left(-v^{2}+\Omega_{2}^{2}+j 2 \zeta_{2} v \Omega_{2}\right)}{\mu \Omega_{2}^{4}} .
\end{aligned}
$$

Let us define the small current detuning as $\gamma=\Omega_{2}-$ $v$. Substituting this into the above formula, we obtain the following expression which is accurate to small terms of the second order:

$$
\begin{aligned}
& \left.T(j v)\right|_{v \approx \Omega_{2}} \\
= & -2 \frac{\left(\Omega_{1}^{2}+j 2 \zeta_{1} \Omega_{2} \Omega_{1}\right)\left(-\Omega_{2} \gamma+j \zeta_{2} \Omega_{2}^{2}\right)}{\mu \Omega_{2}^{4}} .
\end{aligned}
$$

For the magnitude we find: 


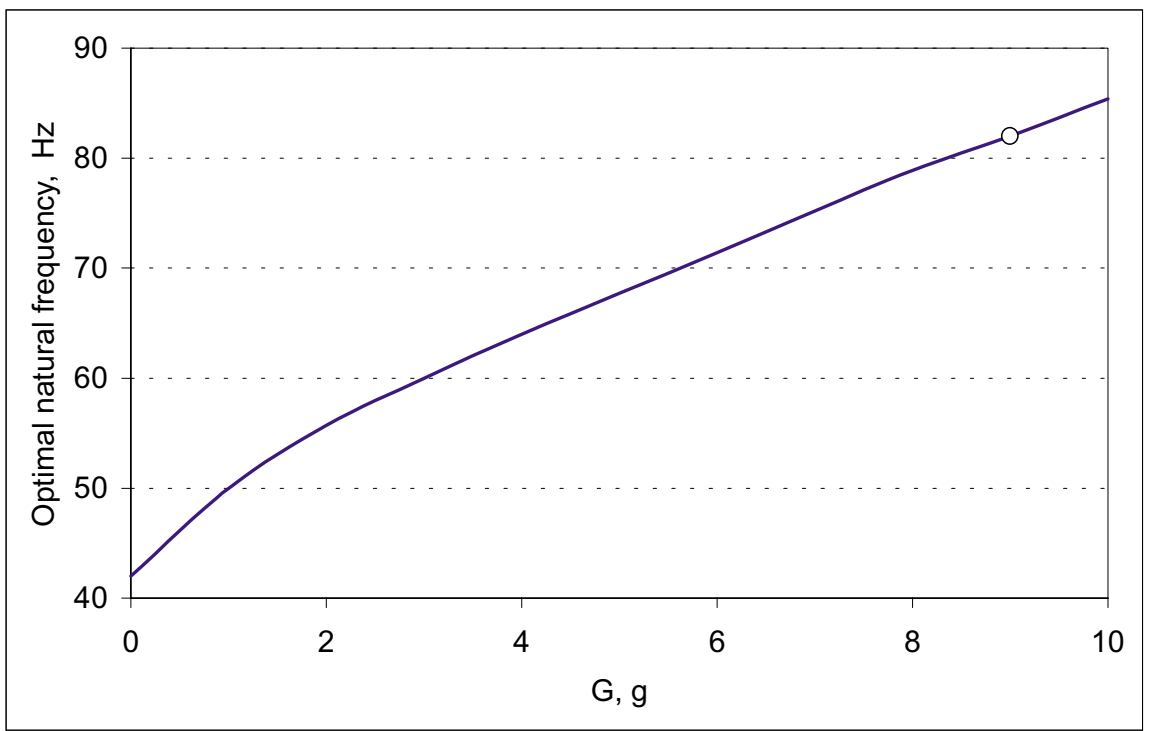

(a)

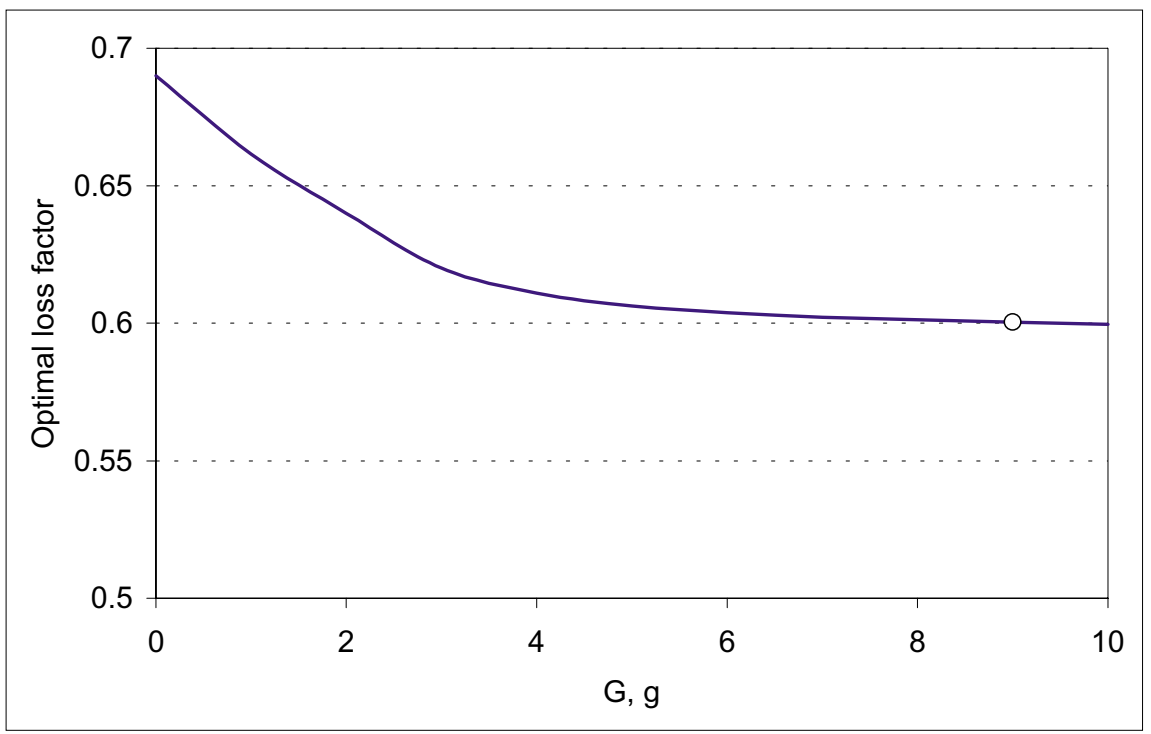

(b)

Fig. 3. Optimal loss factor (a) and natural frequency (b) of the primary suspension at different constant accelerations.

$$
\begin{aligned}
&\left|T\left(\Omega_{2}-\gamma\right)\right|= \frac{2 \sqrt{\zeta_{2}^{2}+\bar{\gamma}^{2}}}{\mu} \frac{\Omega_{1}}{\Omega_{2}} \\
& \sqrt{\left(\frac{\Omega_{1}}{\Omega_{2}}\right)^{2}+4 \zeta_{1}^{2}},
\end{aligned}
$$

where $\bar{\gamma}=\frac{\Omega_{2}-v}{\Omega_{2}}$ denotes the relative detuning.

It can be seen that $\left|T\left(\Omega_{2}-\gamma\right)\right|$ is reciprocal to the mass factor. However, an increase in the mass ratio causes an increase in the variance of relative deflection of the primary system under random excitation, as seen from (6).

It can be also seen that $\left|T\left(\Omega_{2}-\gamma\right)\right|$ increases with the growth of the ratio $\frac{\Omega_{1}}{\Omega_{2}}$, loss factors $\zeta_{1}$ and $\zeta_{2}$. From (9), we also find that $\left|T\left(\Omega_{2}-\gamma\right)\right|$ is the even function of the detuning, $\gamma$, therefore:

$$
\left|T\left[j\left(v_{d}+\Theta\right)\right]\right|=\left|T\left[j\left(v_{d}-\Theta\right)\right]\right| .
$$

From (9), we can define the formula which is used for the practical tuning (see section Industrial case) of 


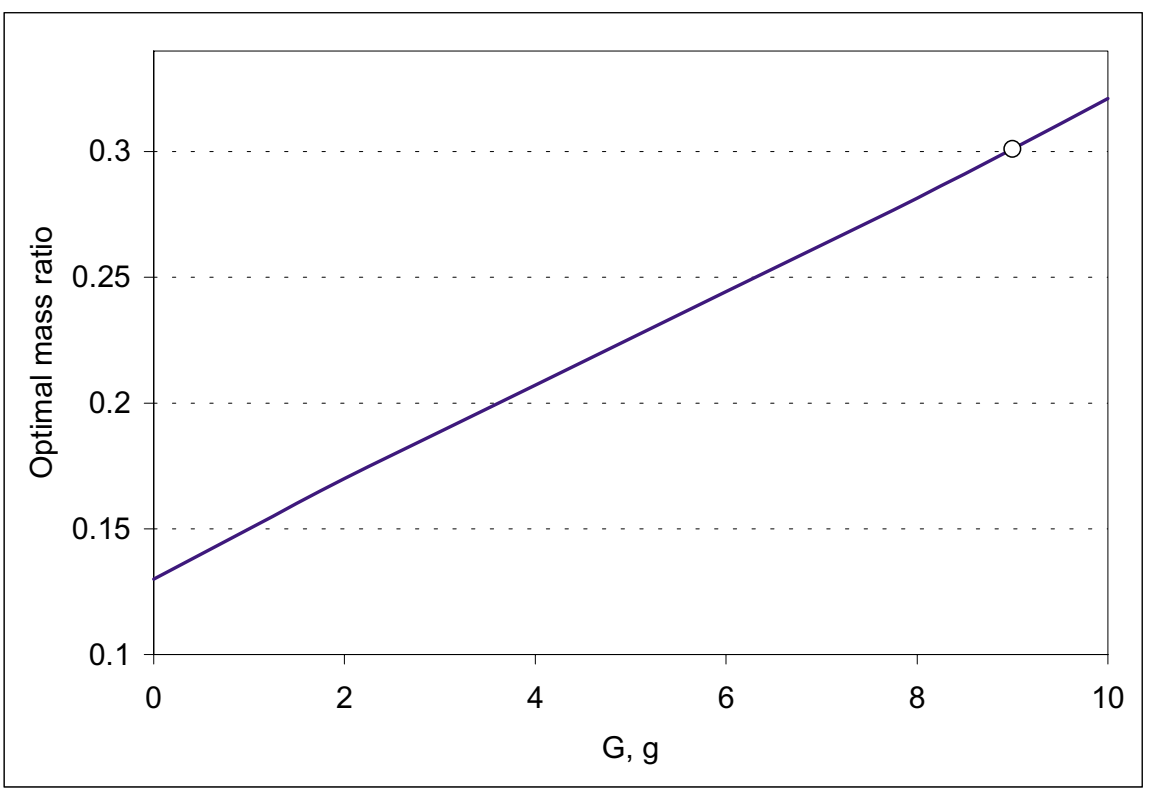

Fig. 4. Optimal mass ratio at different constant accelerations.

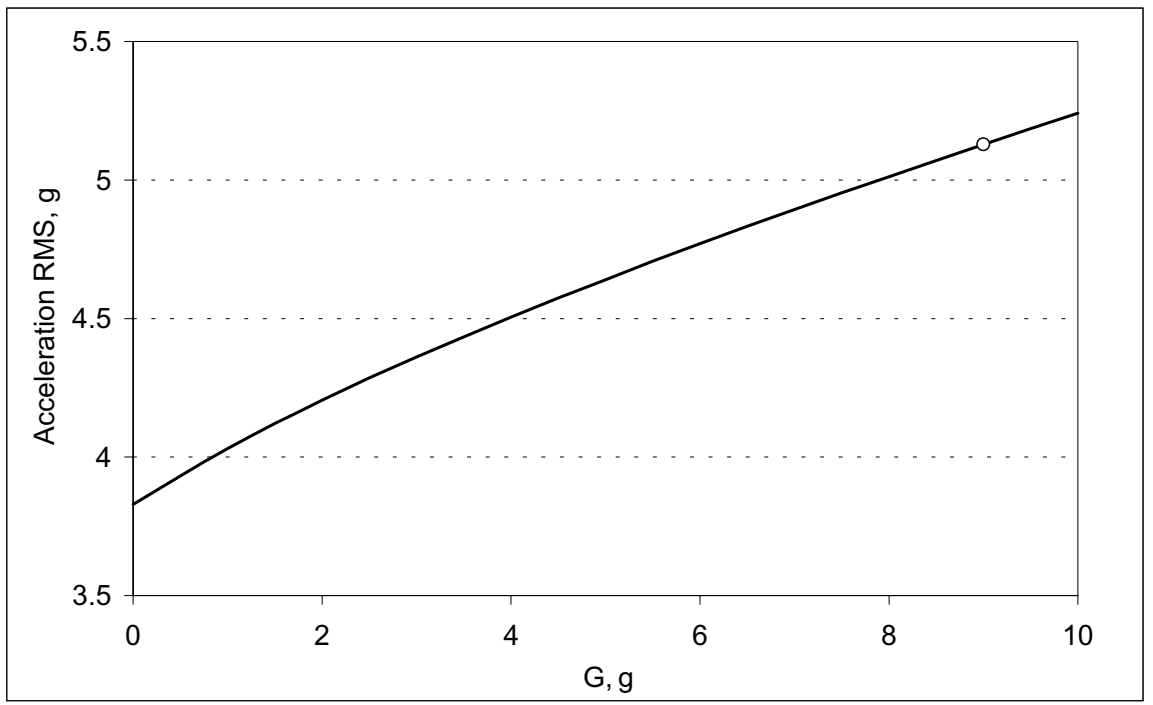

Fig. 5. Optimal values of RMS of acceleration of the primary system at different constant accelerations.

the absorber:

$$
\frac{\left|T\left(\Omega_{2}-\gamma\right)\right|}{\left|T\left(\Omega_{2}\right)\right|}=\sqrt{1+\frac{\bar{\gamma}^{2}}{\zeta_{2}^{2}}}
$$

which indicates what level of vibration suppression is required at the anti-resonant frequency in order to guarantee the desired suppression at given detuning $\bar{\gamma}$ and loss factor $\zeta_{2}$.

\subsection{Optimal design of vibration protection system}

The objective of the optimisation of the vibration protection system is the minimisation of the overall RMS level of acceleration of the primary system subject to constraints of limited relative deflections and desired suppression of the self-induced force.

In applying the $n \sigma$ rule [10] ( $n=3$ is a typical value providing the instantaneous level of acceleration to be less then $3 \sigma$ with the probability of $99.73 \%$ ) to 


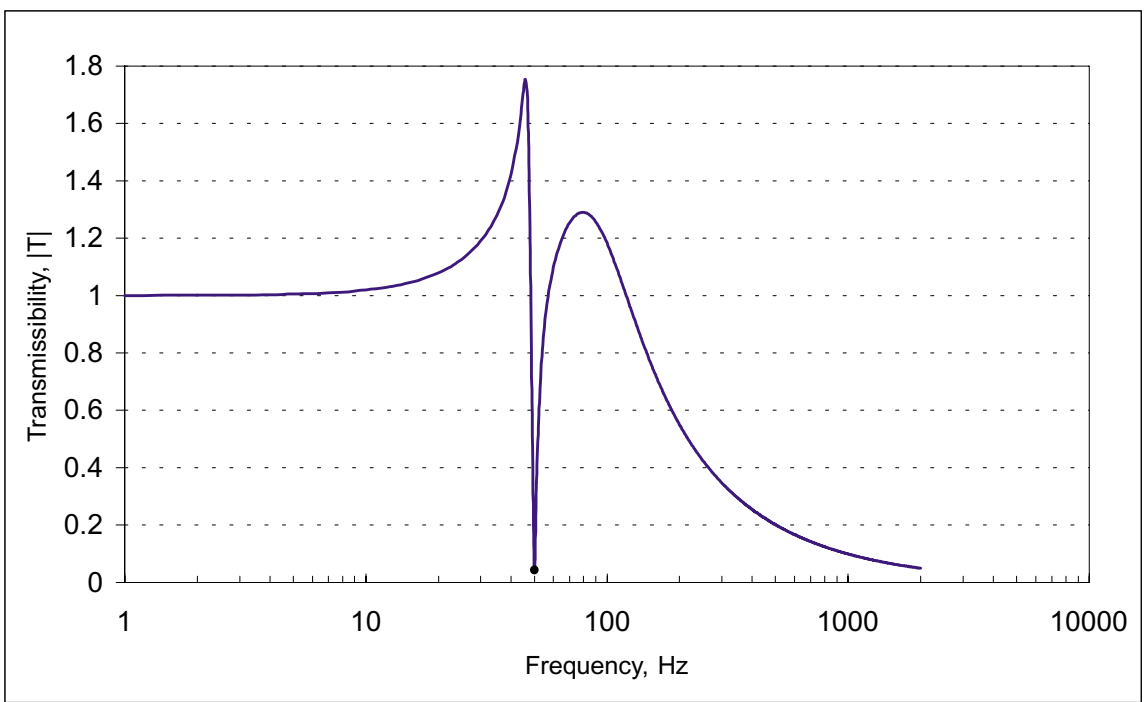

Fig. 6. Absolute transmissibility of the optimised vibration protection system.

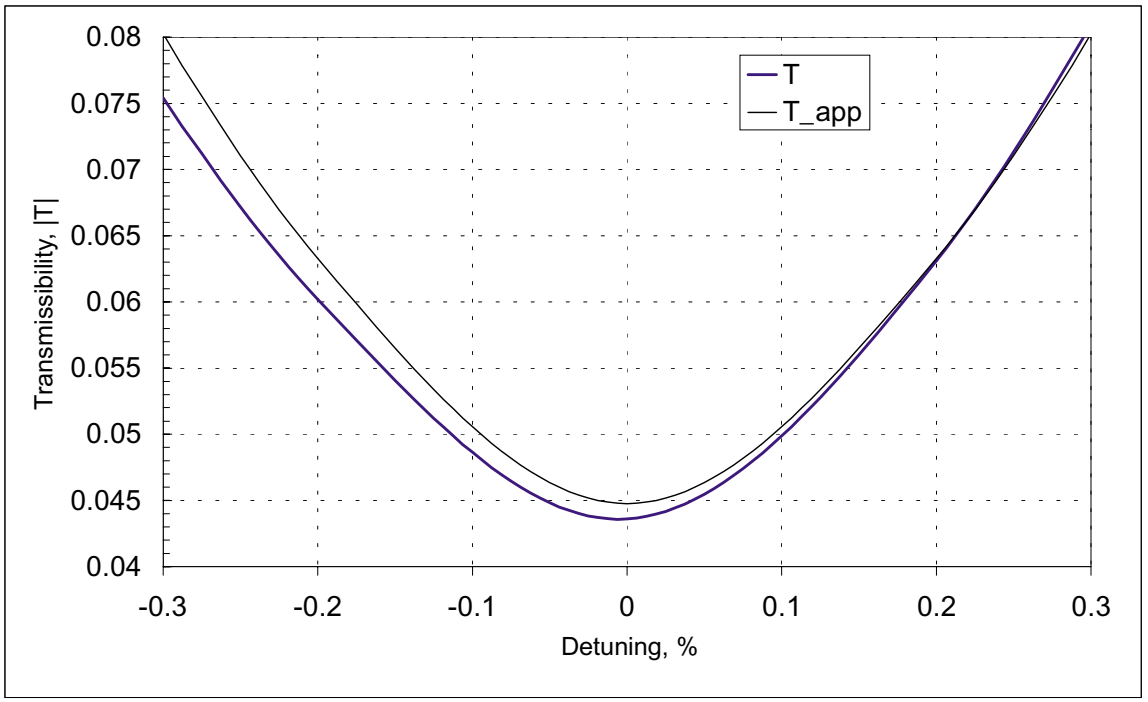

Fig. 7. Absolute transmissibility of the optimised vibration protection system in the vicinity of antiresonance.

the dynamic component of deflection and accounting for the quasi-static relative deflection, the peak relative deflection may be assessed by means of the expression:

$$
\Delta=n \sigma_{X_{1 r e l}}+\lambda
$$

where $\sigma_{X_{1 r e l}}-$ RMS of the relative deflection due to the random base-induced excitation, see Equation (6), $\lambda$ - quasi-static relative deflection due to the action of constant acceleration, see Equation (7).

It is important to note that the component of peak deflection which is due to the action of the self-induced force is not represented in Equation (11). We assume that because of dynamic suppression, this component is negligibly small in comparison with the random and quasi-static components. This assumption may be proven numerically.

Substitution of (6) and (7) into (11) yields the peak deflections of the primary system exposed to random wideband excitation and high g-loading, that is:

$$
\begin{aligned}
\Delta= & n \sqrt{\frac{S_{0}}{4 \Omega_{1}^{3} \zeta_{1}}\left[1+\mu\left(2+\frac{\Omega_{1}^{2}}{\Omega_{2}^{2}}\right)+\mu^{2}\right]} \\
& +\frac{G(1+\mu)}{\Omega_{1}^{2}} .
\end{aligned}
$$




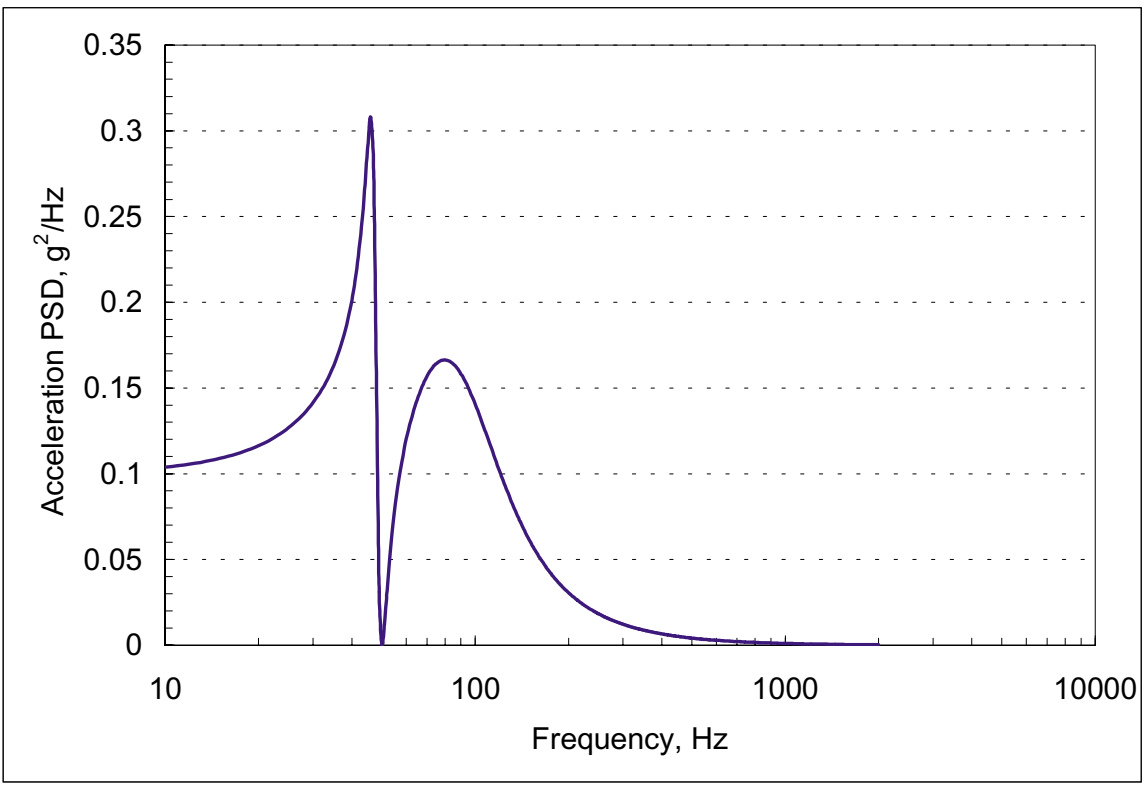

Fig. 8. PSD spectrum of accelerations of the primary system, overall level 4.9g RMS.

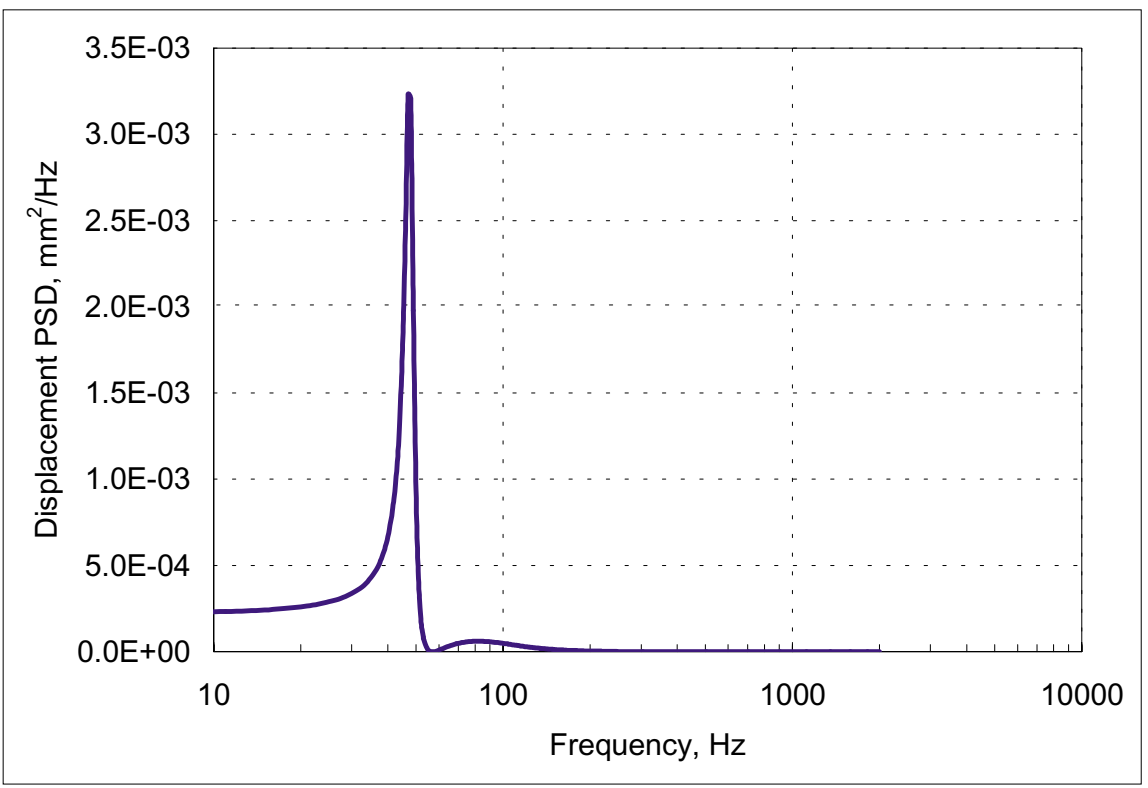

Fig. 9. PSD of relative deflections of the primary system, overall value $0.19 \mathrm{~mm}$ RMS.

Due to the limitation imposed on the peak relative deflection of the primary system we need

$$
\Delta \leqslant \Delta_{0}
$$

where $\Delta_{0}$ is the allowable relative deflection of the primary system. This value is specified by the design of the vibration isolator, gas transfer line, electrical and thermal interfaces, etc.
Similarly, with the help of (9) we impose the next constraint which specifies the desired level of suppression of the self-induced force at given detuning, $\bar{\gamma}$, that is:

$$
T_{0} \leqslant \frac{2 \sqrt{\zeta_{2}^{2}+\bar{\gamma}^{2}}}{\mu} \frac{\Omega_{1}}{\Omega_{2}} \sqrt{\left(\frac{\Omega_{1}}{\Omega_{2}}\right)^{2}+4 \zeta_{1}^{2}} .
$$

Further, by varying the natural frequency and loss 


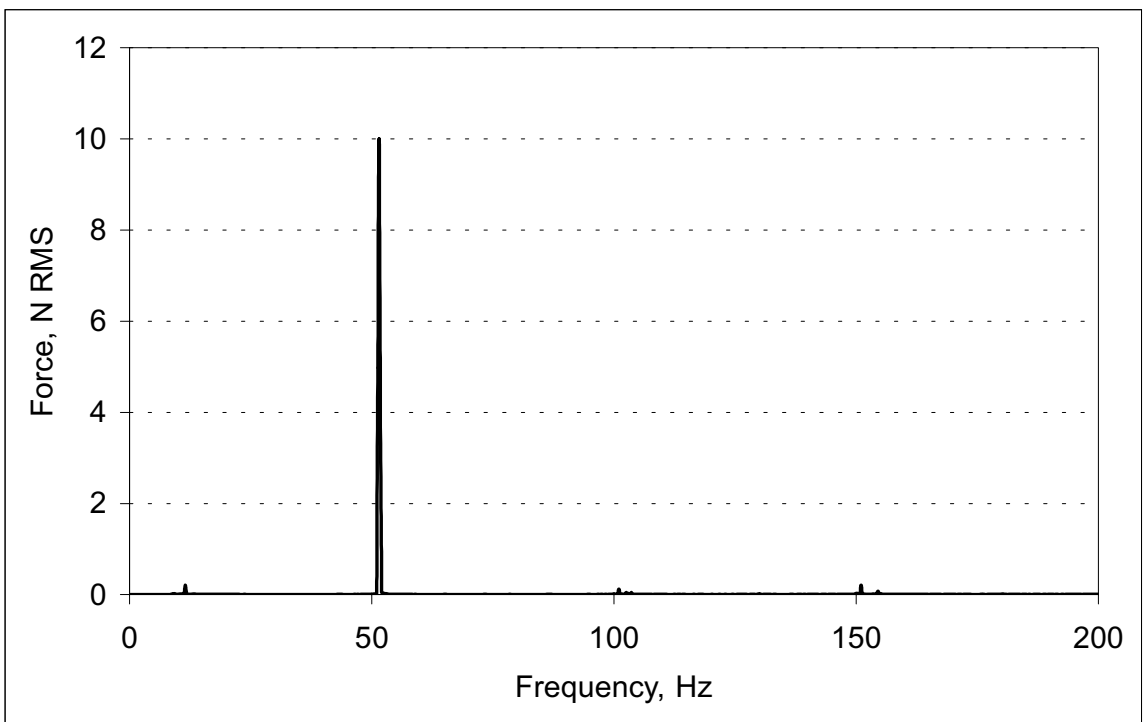

Fig. 10. Spectrum of the self-induced force of the linear compressor; operation under typical heat load at closed loop mode.

factor of the primary suspension and also the mass ratio, we minimise the RMS of acceleration (5) subject to constraints (13) and (14). By so doing, we find successively the optimal set $\Omega_{1}^{*}, \zeta_{1}^{*}$ and $\mu^{*}$.

Consider the example:

$$
\begin{aligned}
& \Delta_{0}=1 \mathrm{~mm} ; S_{0}=0.1 \mathrm{~g}^{2} / \mathrm{Hz} ; \zeta_{2}=0.002 \\
& \bar{\gamma}=0.1 \% ; n=3 ; \frac{\Omega_{2}}{2 \pi}=50 \mathrm{~Hz} ; T_{0}=0.05
\end{aligned}
$$

The value of g-load varies from 0 to $10 \mathrm{~g}$.

The results obtained by application of the numerical procedure are discussed below. Figure 3 shows the required optimum values of natural frequencies (a) and loss factors (b) at different g-loads. From Fig. 3(a), the growth of g-load results in an increase in the optimal natural frequency of the primary suspension, which is required to control the quasi-static component of the relative deflection. It is seen that for constant accelerations above $2 \mathrm{~g}$ the required natural frequency of the primary isolator becomes higher that the driving frequency. From Fig. 3(b), the optimal value of the loss factor decreases slightly with the growth of the g-load.

Figure 4 shows the optimal value of the mass ratio as a function of the considered g-load.

From Fig. 5, the overall RMS level of acceleration of the primary system, calculated by means of formula (5), increases with an increase in the level of considered g-load, but it is still essentially lower that that of the excitation (14g RMS).

The obtained system of vibration protection is capable of effective attenuation of the severity of vibration transmitted to an application through the vibration of the base. At the same time, because of the dynamic de-coupling from the base, such an arrangement becomes suitable for the application of a tuned dynamic absorber and suppression of the self-induced force at a particular anti-resonant frequency.

For instance, in the "worst case" the g-load is 9g. The required optimal vales are $\mu^{*}=30 \% ; \frac{\Omega_{1}}{2 \pi}=83 \mathrm{~Hz}$; $\zeta_{1}^{*}=0.6$. Calculation for the acceleration RMS yields $\sigma_{\ddot{X}_{1}}=5.1 \mathrm{~g}$.

By designing such a system of vibration protection for the worst combination of dynamic and quasi-static loading, we ensure that application will not encounter higher levels of vibration acceleration and peak deflections.

Different approaches to the optimal design of vibration protection systems which accounts for a variety of mission profiles or for the duration of events within each profile may be applied. Such an analysis is beyond the scope of this article.

Figure 6 portrays the graph of absolute transmissibility of the optimised vibration protection system in the frequency range $1-2000 \mathrm{~Hz}$ (the exact expression (8) was used).

Figure 7 shows the transmissibility curve in the close vicinity of the anti-resonance as a function of the small detuning $\bar{\gamma}$ in \% (labelled as $\mathrm{T}$ ). The numerical values of parameters are as specified above, and the exact expression (8) is used. For comparison, the approximate curve is superimposed (labelled as T_app) for the same numerical values (approximate expression (9) is used). 


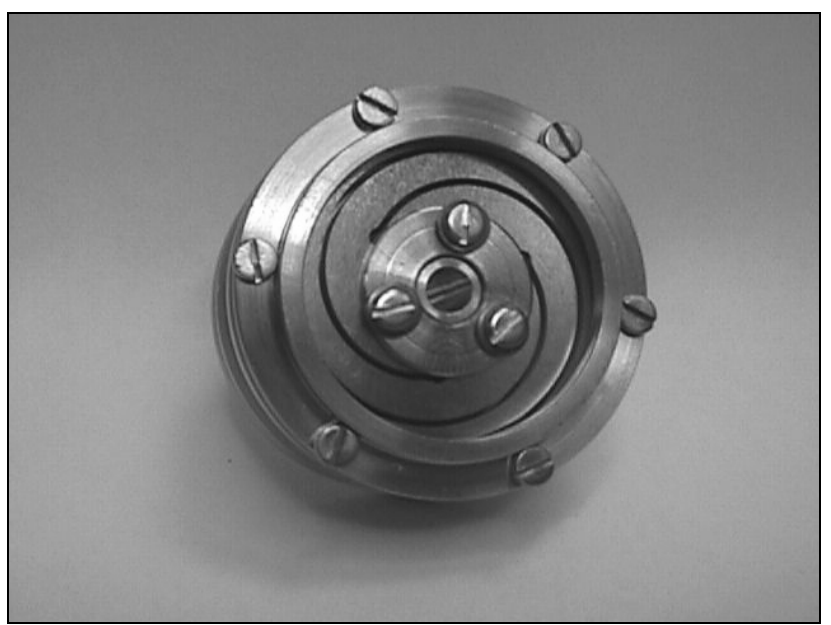

a)

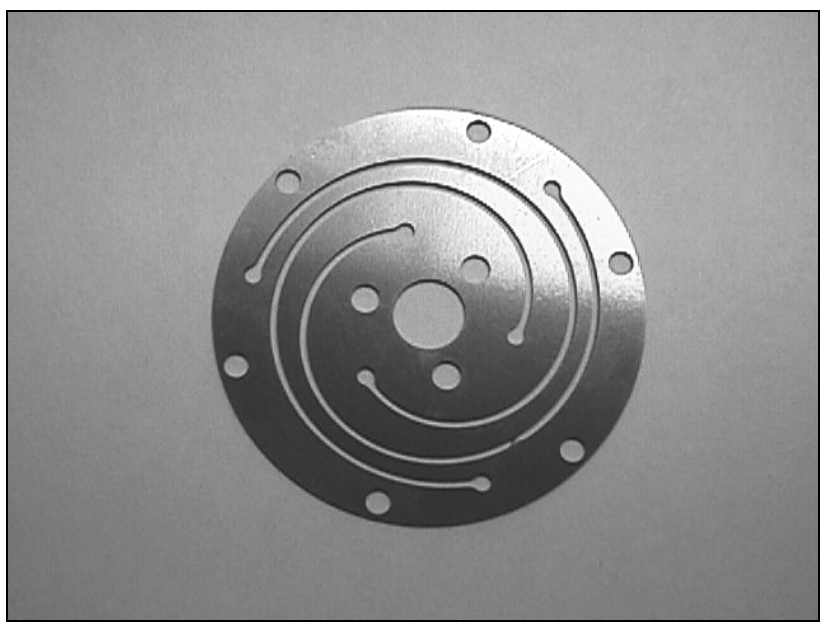

b)

Fig. 11. Picture of dynamic absorber (a) and "Oxford" type flexural element (b).

This shows the fair accuracy of approximate expression (9) which is used in the optimisation procedure.

It is seen that at detuning of $0.1 \%$ the suppression is $|T| \leqslant 0.05$ (20 times attenuation). From formula (10), the required value of the transmissibility at the antiresonant frequency is 0.045 (compare with Fig. 7).

Figure 8 portrays the PSD spectrum of acceleration of the primary system (overall level of $4.9 \mathrm{~g} \mathrm{RMS}$ ). Figure 9 shows the PSD of relative deflections of the primary system.

To determine the sensitivity of the dynamic response of the primary system to the isolator properties, a preliminary analysis is carried out. It was shown numerically that small variations (up to $10 \%$ ) in the values of loss factor and natural frequency do not considerable affect the performance of the vibration protection sys- tem. The detailed analysis of sensitivity is beyond the scope of this article.

\section{Industrial case}

The obtained results were used for design the of a vibration protection system for a particular IR application which relies on the RICOR split Stirling cryogenic cooler K529, as shown in Fig. 1. The selfinduced force, which is produced by the linear compressor, comprises the single-frequency component corresponding to the driving frequency (see spectrum in Fig. 10). The self-induced force, IONRMS@ $@ 50 \mathrm{~Hz}$ was measured when the cryogenic cooler operated in a closed-loop mode under a typical heat load. The Kistler 


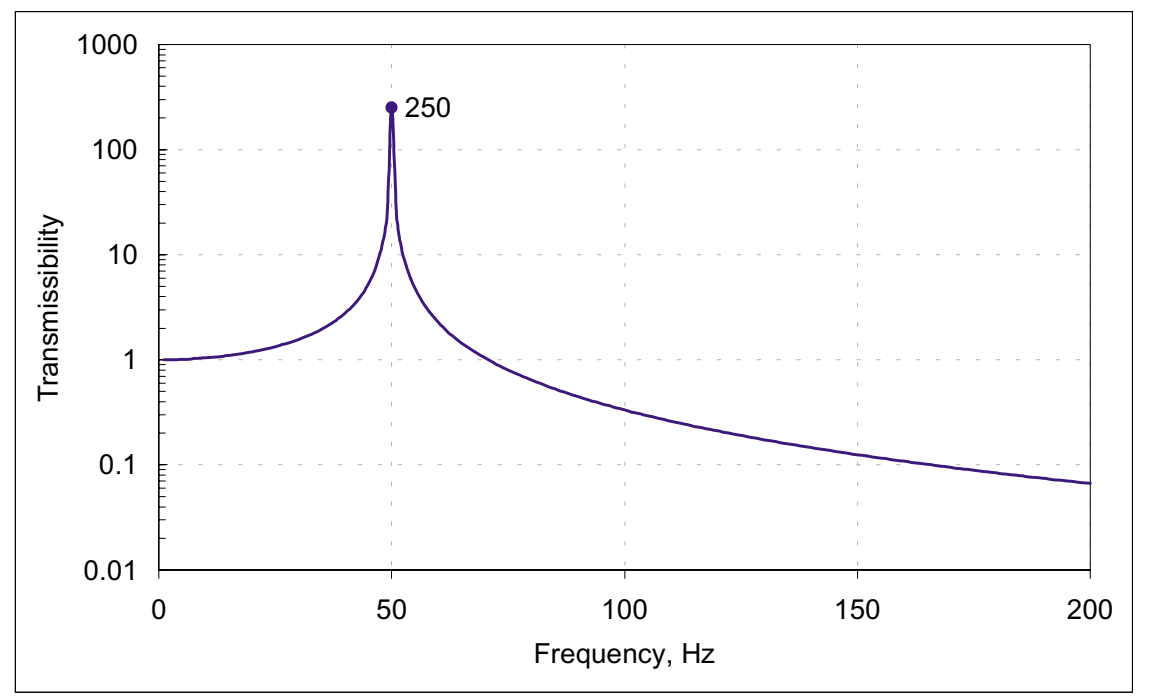

Fig. 12. Absolute transmissibility of absorber.

four-axis dynamometer Type 9272A and Data Physics SignalCalc ACE Dynamic Signal Analyser were used for measurements. This particular component of the self-induced force appears to be the dominant disturbing factor of the specific IR application. The customer specified the allowable force export to be $0.5 \mathrm{~N}$ RMS@50Hz, as required for the smooth operation of the entire application.

The environmental conditions were specified as follows:

- Random vibration: flat PSD $0.1 \mathrm{~g}^{2} / \mathrm{Hz}$ in the frequency range $10-2000 \mathrm{~Hz}$.

- g-load: $9 \mathrm{~g}$.

- Allowable peak relative deflection of linear compressor: $\pm 1 \mathrm{~mm}$.

The dynamic absorber was designed to have a mass of $0.15 \mathrm{~kg}$ providing the required mass ratio of $30 \%$ (see Fig. 11(a) and Fig. 13(b)).

To maintain the low loss factor of the auxiliary system and to reduce the temperature sensitivity and residual stresses, the flexural elements are designed as allmetal "Oxford" type flat springs and manufactured by photo-hatching of the hardened stainless steel SST 304 sheets of thickness $0.2 \mathrm{~mm}$ (see Fig. 11(b)). The geometry of the flexural elements is optimised to ensure linearity and fail-safe operation within the range of dynamic damper deflection $\pm 1 \mathrm{~mm}$. As a result of the careful design, a loss factor of $0.2 \%$ is achieved. The experimentally measured transmissibility of the dynamic absorber in Fig. 12 shows amplification of 250 at resonance. The coarse tuning of the natural fre- quency of an absorber to $50 \pm 0.125 \mathrm{~Hz}$ involves measurement of its resonant frequency and fine adjustment of its mass. Such an accuracy is provided by the resolution of the Data Physics SignalCalc ACE Dynamic Signal Analyser which was used in this measurements.

Figure 13 portrays the schematic (a) and photo (b) of the experimental rig. The rig contains linear compressor (1)(RICOR split Stirling linear cryogenic cooler model K529) mounted by means of the all-metal flexural elements (2) over the rigid frame (3). The two viscoelastic O-rings (4) are used to provide for the necessary stiffness and damping. They are manufactured of the thermoplastic material ISODAMP C-1000 Series (E-A-R, Specialty Composites). The primary suspension described is designed to have the desired natural frequency of $83 \mathrm{~Hz}$ and loss factor of 0.6 , see the measured absolute transmissibility in Fig. 14. The procedure of this measurement involves mounting of the experimental rig upon the sensitive surface of the dynamometer, application of the testing impact force to the compressor housing by means of the Bruel and Kjaer Impact Hammer Type 8202 and measuring the appropriate transfer function.

The combination of all-metal flexural elements (2) and highly damped visco-elastic O-rings (4) is used for the greater ease of manipulation in experiments of the elastic and damping properties of the primary suspension. The final design eventually requires a simple, less expensive and more reliable solution such as metal mesh bushings the mechanical properties of which are practically invariable within the temperature range encountered $\left(-40^{\circ} \mathrm{C}\right.$ to $+70^{\circ} \mathrm{C}$, typically). 


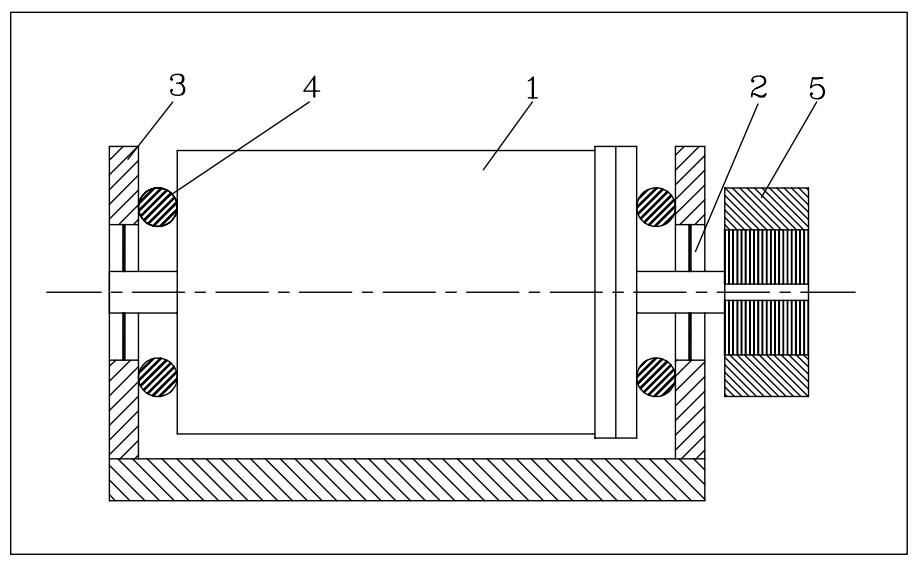

(a)

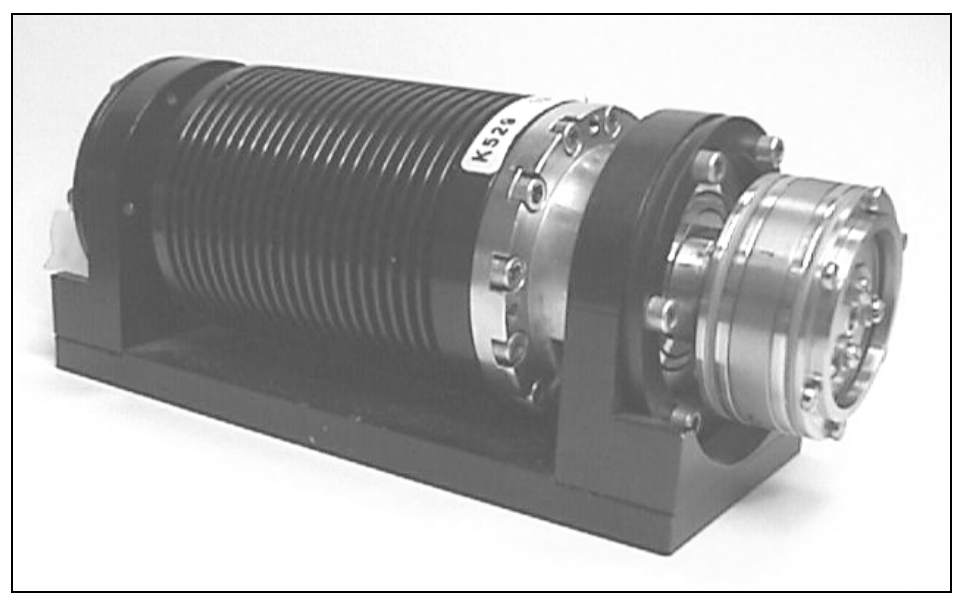

(b)

Fig. 13. Schematic (a) and photo (b) of the vibration protection system which combines stiff and heavily damped vibration isolator and tuned vibration absorber.

The dynamic absorber (5) is then affixed to the charge port of the compressor collinear to the motor axis. By combining the coarse tuning of the natural frequency of the absorber (as described above) and fine-tuning of the controller driving frequency, the required vibration suppression is achieved (see spectrum of residual force export in Fig. 15). It is important to note that the cryogenic cooler's enhanced digital controller is designed to be capable of precise and easy adjustment and maintenance of the driving frequency with an accuracy of better than $0.02 \%( \pm 0.01 \mathrm{~Hz})$.

At anti-resonant frequency, we have the minimum force export of $0.45 \mathrm{~N}$ RMS@50Hz. By changing the driving frequency by $0.1 \mathrm{~Hz}$ from the actual antiresonant frequency, we obtaine an increase in the force export to lower than the desired 0.5N RMS@50Hz. These values are in fair agreement with formula (10).

\subsection{Random vibration test}

The above vibration protection system is subjected to the random vibration test with flat PSD $0.1 \mathrm{~g}^{2} / \mathrm{Hz}$ in the frequency range $10-2000 \mathrm{~Hz}$ (14 g RMS overall level).

The vibration test system V550/PA550L (Ling Dynamic Systems Ltd) was used in this experiment.

Figure 16 shows the spectrum of the PSD of acceleration which is measured on the compressor housing. The overall RMS level is measured as $5 \mathrm{~g}$ RMS (compare with predicted $4.9 \mathrm{~g}$ RMS).

The graph of the PSD of relative deflections of the primary system (see Fig. 17) is obtained by means of measuring the difference between the signals of two accelerometers, mounted on the compressor and on the shaker, and successive double integrating. Correspond- 


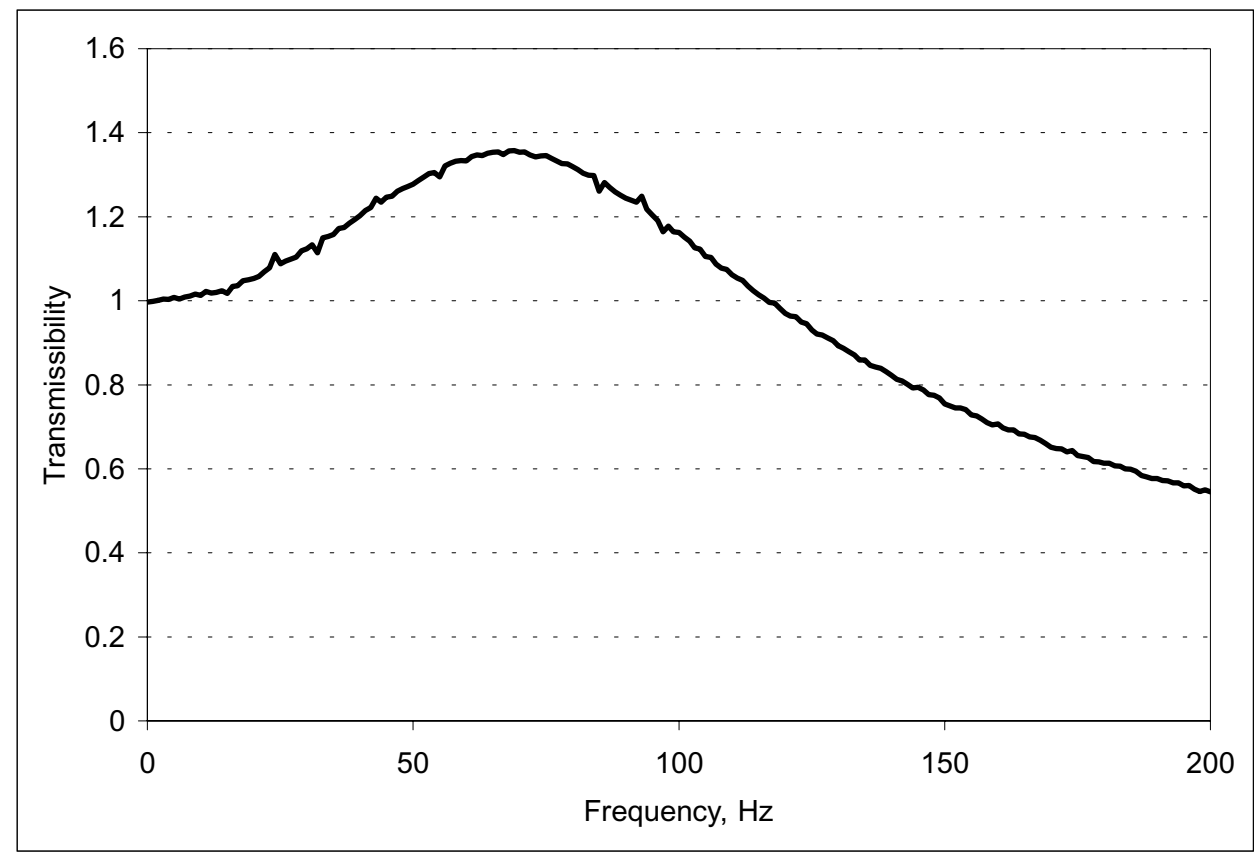

Fig. 14. Absolute transmissibility of the primary system.

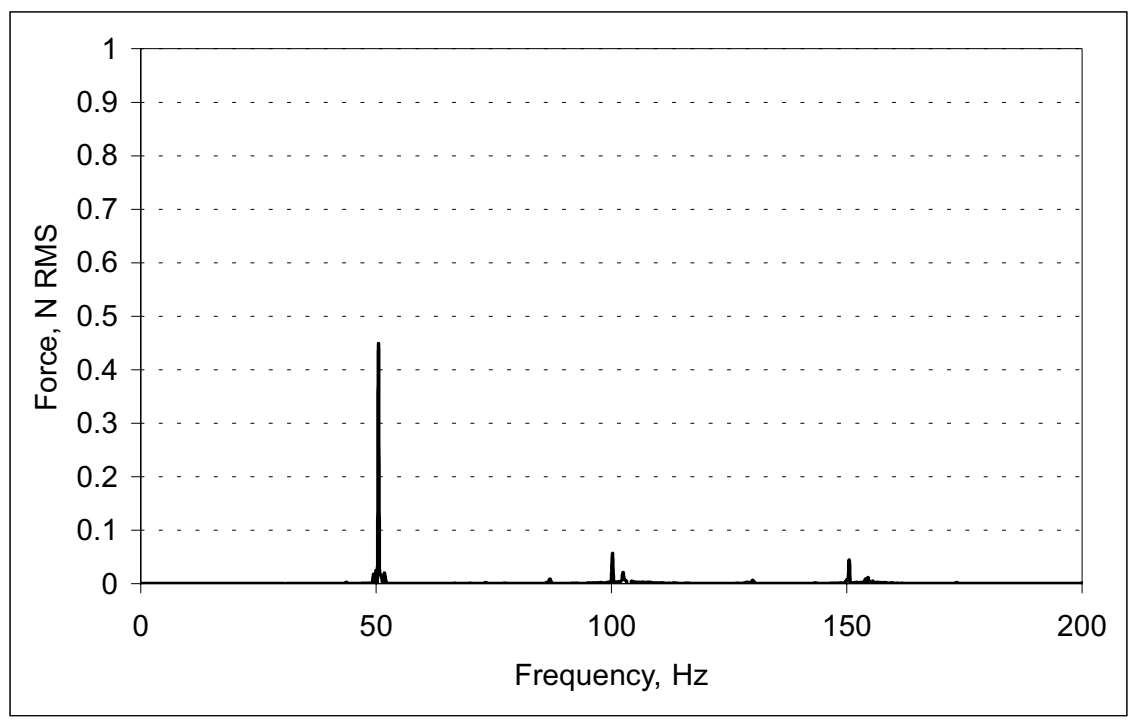

Fig. 15. Spectrum of residual self-induced force.

ing calculation yields $0.18 \mathrm{~mm}$ RMS for the overall RMS of the relative deflection (compare with predicted 0.19 mm RMS).

\section{Conclusions}

The dynamics of the vibration protection system which relies on the stiff and heavily damped vibration isolator in combination with an undamped tuned vibration absorber, was studied for use in the design of the particular IR device comprising RICOR linear split Stirling cryogenic cooler model K529.

The analytical solution showed that an optimally designed system successfully combines the properties of the desired wideband attenuation of the base induced random vibration and close narrowband suppression of 


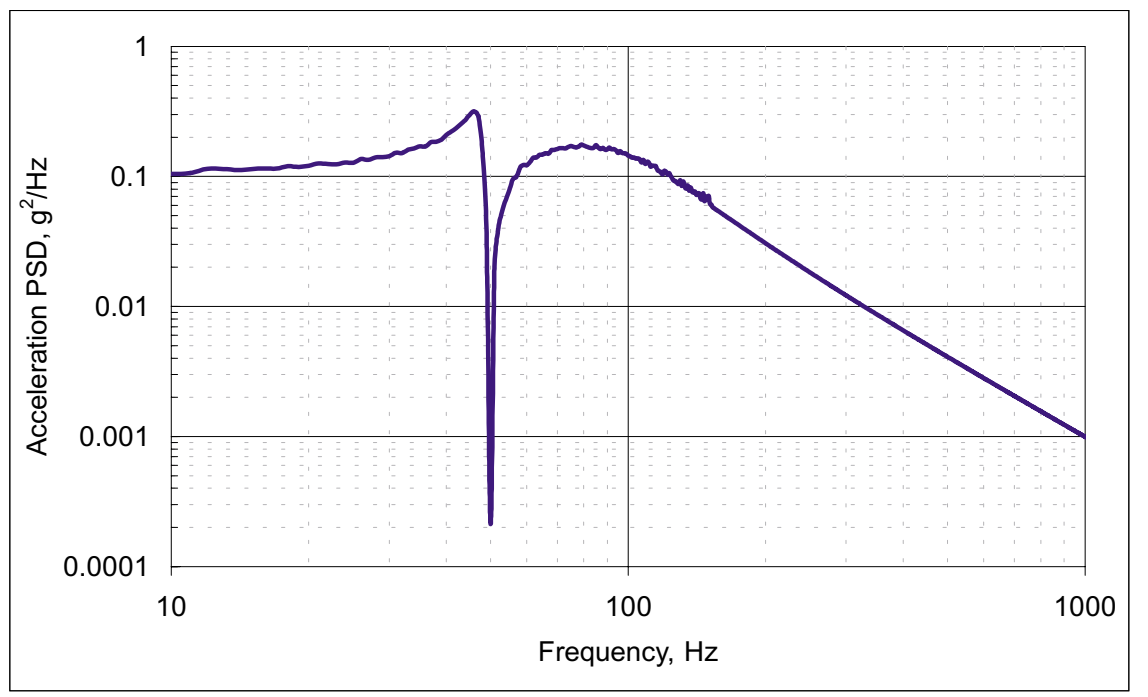

Fig. 16. Spectrum of acceleration PSD of the primary system under wideband random excitation.

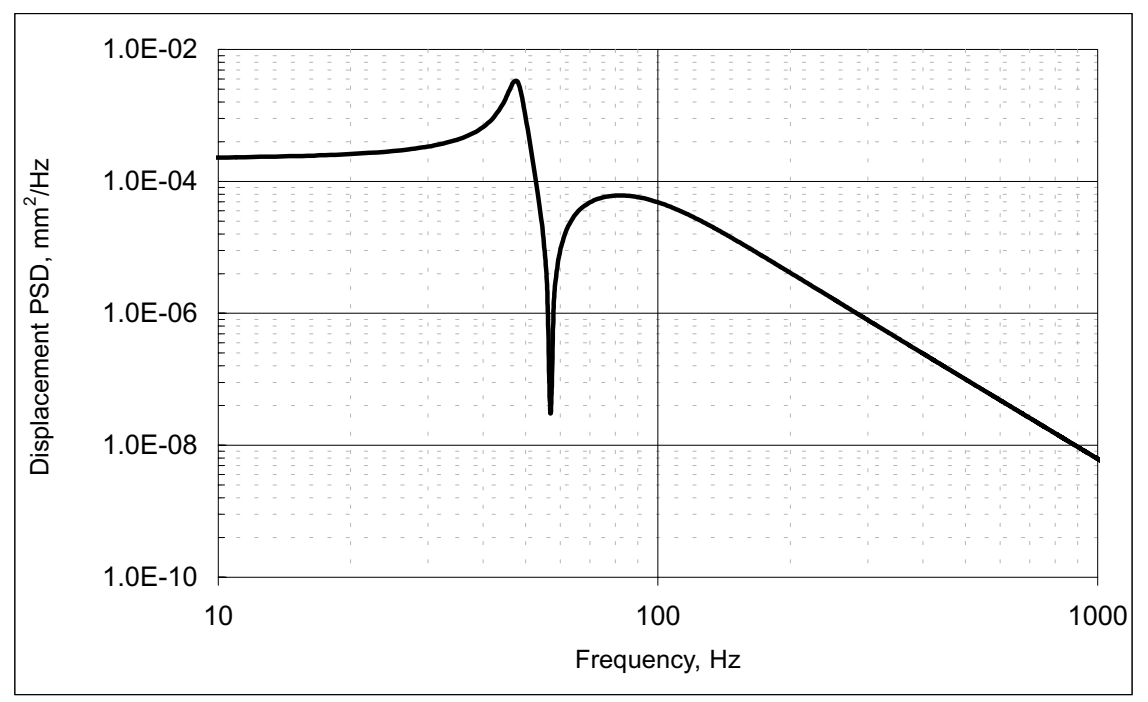

Fig. 17. Spectrum of relative deflection PSD of the primary system under wideband random excitation.

the harmonic self-induced force within restrained peak deflections of the linear compressor.

The results of the calculations were in a fair agreement with the experimental testing. The experimentally tested system of vibration protection demonstrated attenuation by a factor of 3 of the overall level of baseinduced random vibration, and suppression of the selfinduced force by a factor of 20 .

\section{References}

[1] V.I. Babitsky and A.M. Veprik, Smart nonlinear structure of vibration isolation, Proceedings of the 5th International
Congress on Sound and Vibration, Adelaide, Australia, 1997, pp. 2971-2980.

[2] V.I. Babitsky and A.M. Veprik, Universal bumpered vibration isolator for severe environment, Journal of Sound and Vibration 218(2) (1998).

[3] L.A. Coleman, Infrared Sensors: The Eyes of the Digital Battlefield, Military \& Aerospace Electronics, July 1994, pp. 2931.

[4] S.H. Crandall and W.D. Mark, Random vibration in mechanical systems, Academic Press, NY, 1963.

[5] J.L. Miller, Principles of infrared technology, A practical guide to the state of the art, Van Nostrand Reinhold, 1994.

[6] MIL-STD-810E Military Standard, Environmental Test Methods, Department of Defence, Washington, DC, 1988. 
[7] J. Ormondroyd and J.P. Den Hartog, The theory of the dynamic vibration absorber, Transactions of ASME $\mathbf{5 0}$ (1928).

[8] A.M. Veprik, A. Meromi and A. Leshecz, Novel technique of vibration control for split Stirling cryogenic cooler with linear compressor, Proceedings of SPIE's 11th Annual International Symposium on Aerospace/Defence Sensing, Simulation and Controls "AeroSense, Orlando, Florida, Vol. 3061, Infrared Technology and Applications XXIII, 1995, pp. 640-651.
[9] J.C. Snowdon, Vibration and shock in damped mechanical systems, John Wiley, NY, 1968.

[10] J.L. Sloan, Design and Packaging of Electronic Equipment, Van Nostrand Reinhold Company, 1985.

[11] Shock and Vibration Handbook, C.M. Harris and C.E. Crede, eds., McGraw-Hill Book Company, New York, 1976.

[12] G. Walker, Cryogenic coolers, Part2 - Applications, Plenum Press, New York, 1983. 

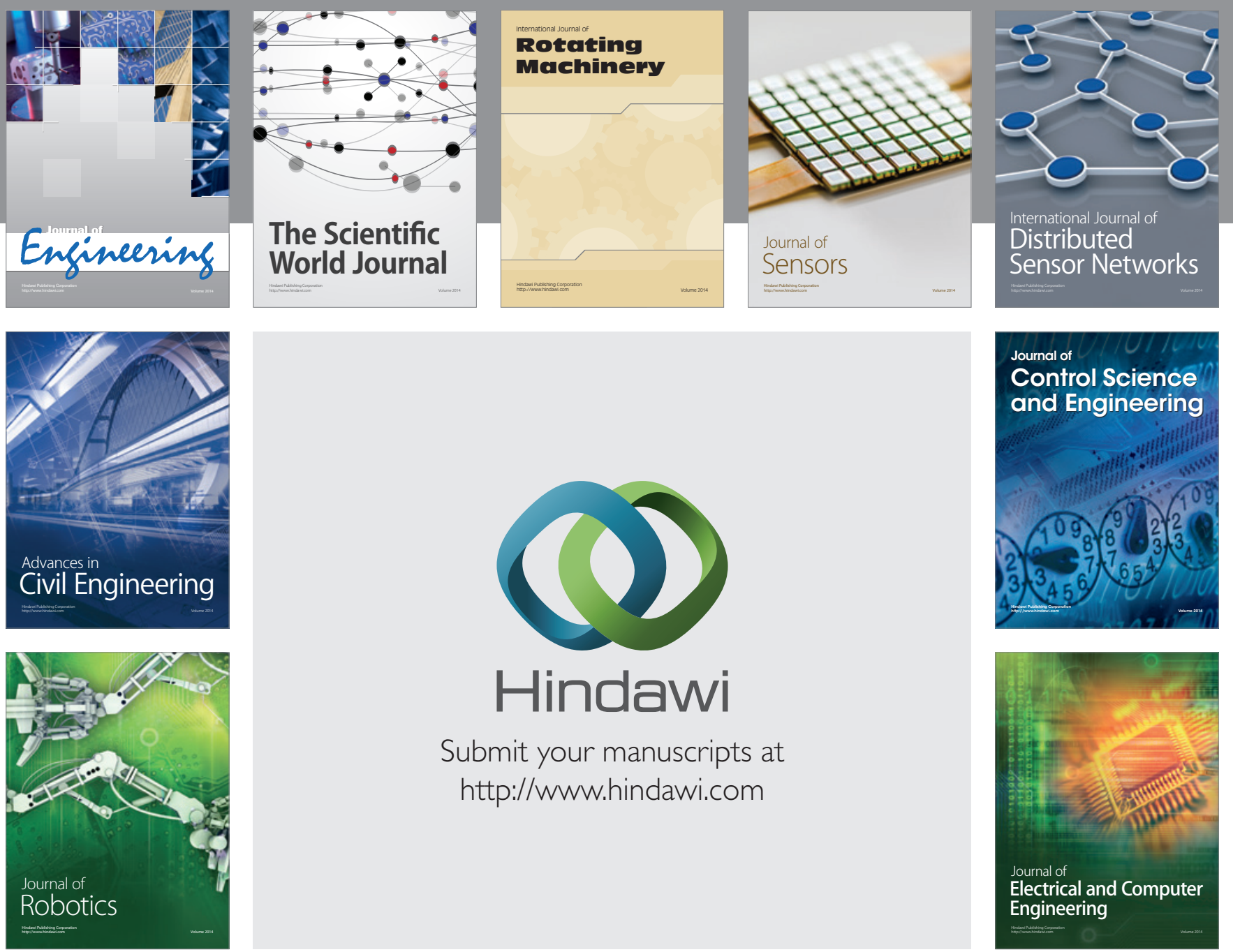

Submit your manuscripts at

http://www.hindawi.com
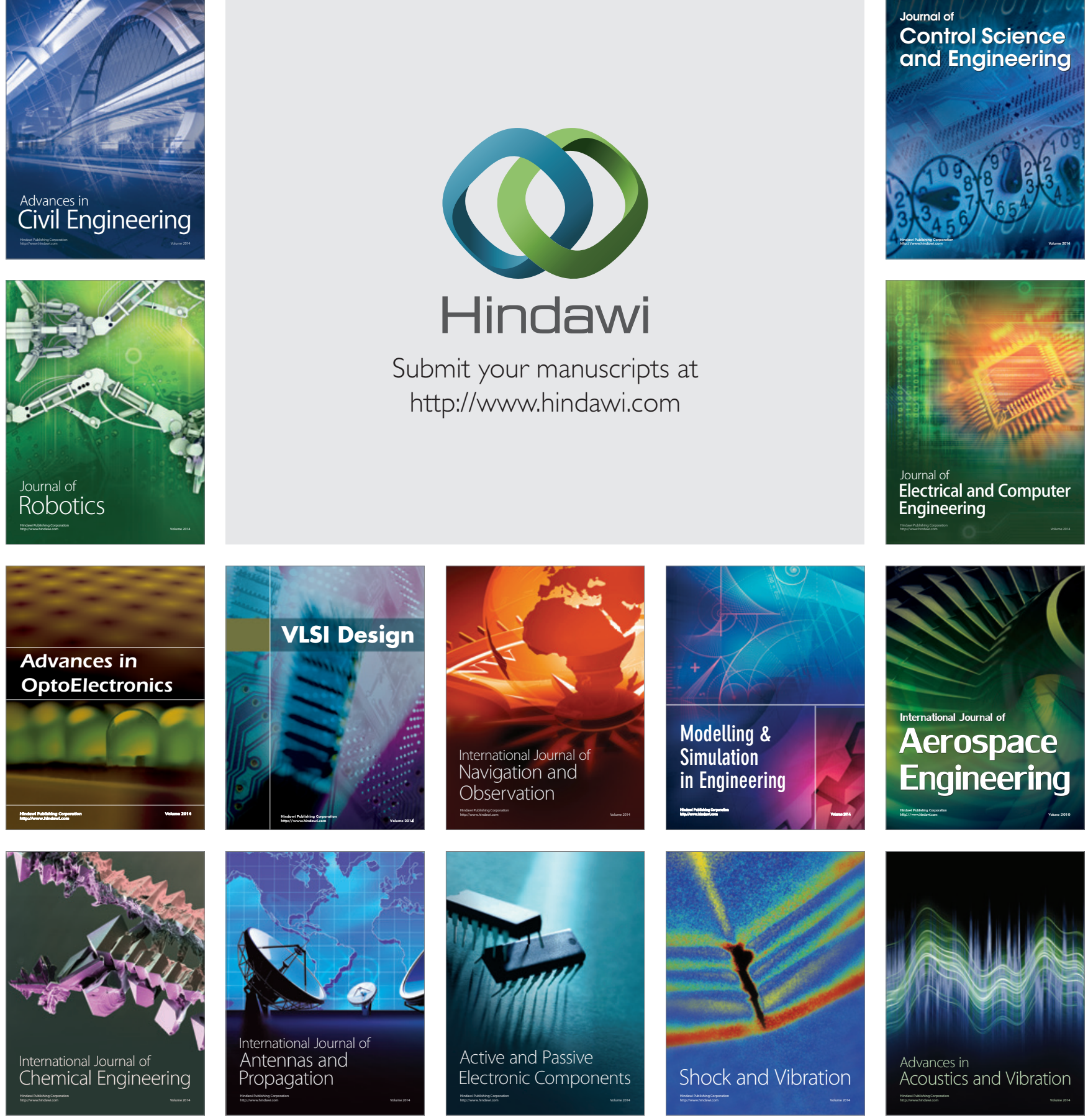